\title{
Rutin, quercetin, and free amino acid analysis in buckwheat (Fagopyrum) seeds from different locations
}

\author{
C.Z. Bai' ${ }^{1}$, M.L. Feng' ${ }^{1}$, X.L. Hao', Q.M. Zhong' ${ }^{1}$, L.G. Tong ${ }^{1}$ and Z.H. Wang ${ }^{2}$ \\ ${ }^{1}$ Central Laboratory, Chinese Medicine Hospital of Shanxi Province, Taiyuan, China \\ ${ }^{2}$ Key Laboratory for Chemical Biology and Molecular Engineering of Ministry of \\ Education, Institute of Biotechnology, Shanxi University, Taiyuan, China \\ Corresponding author: Z.H. Wang \\ E-mail: wnagzhuanhua_hh@163.com \\ Genet. Mol. Res. 14 (4): 19040-19048 (2015) \\ Received August 15, 2015 \\ Accepted October 16, 2015 \\ Published December 29, 2015 \\ DOI http://dx.doi.org/10.4238/2015.December.29.11
}

ABSTRACT. In this study, five common buckwheats and nine tartary buckwheats grown at different locations were analyzed for the contents of rutin, quercetin, and amino acids by high-performance liquid chromatography and spectrophotometry. The rutin content was higher than quercetin in buckwheat seeds. Rutin content was in the range from 0.05 ( $0.05 \mathrm{~g}$ per $100 \mathrm{~g}$ dry seeds) to $1.35 \%$ of buckwheat seeds. Quercetin content varied from 0.01 to $0.17 \%$ and in some common buckwheats it was even difficult to detect. Comparatively, tartary buckwheat seeds contained more rutin and quercetin than common buckwheat seeds. Meanwhile, the bran has higher rutin content than the farina in tartary buckwheat seeds, with a respective content of 0.45 to $1.19 \%$ and 0.14 to $0.67 \%$. It was found that amino acid contents were around 1.79 to $12.65 \%$ (farina) and 5.74 to $7.89 \%$ (bran) in common buckwheats, and 1.73 to $5.63 \%$ (farina) and 2.64 to $16.78 \%$ (bran) in tartary buckwheat seeds. The highest total rutin content was found to be $1.35 \%$ in tartary buckwheat seeds from Sichuan, China. The highest total amounts of amino acid were detected to be $20.13 \%$ in tartary buckwheat seeds from Changzhi, Shanxi Province (China). Our 
results suggested that food products made of whole-buckwheat flour are healthier than those made of fine white flour.

Key words: Buckwheat; Farina; Bran; Rutin; Quercetin; Amino acid

\section{INTRODUCTION}

Buckwheat, which belongs to the family Polygonaceae, genus Fagopyrum, has been a popular health food in Asian and European countries for a long time. The most widely grown buckwheat species include common buckwheat (Fagopyrum esculentum) and tartary buckwheat (Fagopyrum tataricum) (Zhang et al., 2012). Buckwheat is a unique dual-use cereal crop and has attracted more and more attention due to both its nutritional and medicinal values in recent years (Guo et al., 2011). Buckwheat contains many important bioactive compounds such as protein rich in essential amino acids, oil rich in essential fatty acids (Horbowicz and Obendorf, 1992; Steadman et al., 2001a), starch with a low glycemic index, polyphenol compounds (including rutin, quercetin, orientin, vitexin, isovitexin and isoorientin) (Danihelová and Šturdík, 2012; Sharma et al., 2012), and many essential minerals (Steadman et al., 2001b). Studies have revealed that buckwheat can cure chronic human diseases, decrease blood cholesterin, inhibit mammary cancer, prevent gallstones and many others (Tomotake et al., 2000). As a result, buckwheat has been listed as health protection food in many countries, and many nutritional meals related to buckwheat have been developed, such as buckwheat bread, noodles, soft drinks, beverages, tea, and buckwheat sprouts (Xiao, 2003).

Although a natural vasorelaxant compound was identified from rutin-free tartary buckwheat extracted by Matsui et al. (2010), it is well known that the many unique physiological functions of buckwheat are mainly attributed to its high content of flavonoids, especially rutin (Jiang et al., 2007). Quercetin is an important factor for plant secondary metabolism; it has a wide range of biological activity and is also a promising candidate for prevention and treatment of various cancers (Joanna et al., 2011; Wang et al., 2012). The quercetin glycosides and free quercetin in buckwheat flowers, leaves, stems, and achenes were discovered by Dadakova in 2010. Plant polyphenol content is influenced by many factors such as variety, location, harvest month and others (Zou et al., 2012; Gunaratne et al., 2013; Zhang et al., 2013). Meanwhile, the phenolic contents may vary in different tissues (Pugliese et al., 2013). Amino acid content is an important factor to evaluate nutritional value. Many plants have been investigated for their content or biodiversity of free amino acids (Pratta et al., 2011; Elfalleh et al., 2012). However, little is known about the comprehensive assessment of buckwheat seeds based on the contents of rutin, quercetin, and free amino acids. In order to determine these beneficial components in buckwheat seeds, fourteen samples collected from different locations were studied by means of spectrophotometry and HPLC in the present study. The results may guide people to choose appropriate material (buckwheat fine flour or whole flour) and food processes.

\section{MATERIAL AND METHODS}

\section{Apparatus and materials}

High-performance liquid chromatography (HPLC; HITACHI, Japan), Labsystems Multiskam MK3 ELISA (Thermo, USA), Vacuum Filter (Waters, USA). 
All reagents used for HPLC and LC-MS were of HPLC purity (Tianjin Secondary Factory for Chemical Agent, China). The other chemicals were of analytical grade and were filtered with a vacuum through a $0.45-\mu \mathrm{m}$ membrane (Millipore, USA). Standards of rutin (95\%) and quercetin were purchased from Sigma (USA). Amino Acid Quantitation Kit was purchased from Nanjing Jiancheng Biological Ltd. (China).

\section{Sample preparation}

Buckwheat grains harvested in 2009 were provided by the 11th International Symposium of Buckwheat. The plant materials were dried and stored under refrigerated conditions. Common buckwheat from five locations and tartary buckwheat from nine locations were used (Table 1). Hulled buckwheat seeds were ground and then sieved with a number 40 mesh screen in order to obtain farina and bran samples. Accurately weighed farina and bran samples were defatted in Soxhlet extraction with diethyl ether as the extraction agent for $40 \mathrm{~min}$. Prepared samples were then extracted with $40 \mathrm{~mL} 95 \%$ ethanol at $80^{\circ} \mathrm{C}$ for $4 \mathrm{~h}$. After concentration, the extraction was transferred into a $10 \mathrm{~mL}$ measuring flask and filtered through a cellulose acetate filter $(0.45 \mu \mathrm{m}$; Millipore, USA) for quantification.

\begin{tabular}{|c|c|c|c|c|c|c|c|c|}
\hline Sample & 1 & 2 & 3 & 4 & 5 & 6 & 7 & 8 \\
\hline Cultivar & $\mathrm{CB}$ & $\mathrm{CB}$ & $\mathrm{CB}$ & $\mathrm{CB}$ & $\mathrm{CB}$ & TB & TB & TB \\
\hline Location & Hebei, China & Yulin, Shanxi, China & Taiyuan, Shanxi, China & Czech & German & Bhutan1 & Mexico & German \\
\hline Sample & 9 & 10 & 11 & 12 & 13 & 14 & & \\
\hline Cultivar & TB & TB & TB & TB & TB & TB & & \\
\hline Location & US & Jiangxi, China & Changzhi, Shanxi, China & Guizhou, China & Sichuan, China & Yunnan, China & & \\
\hline
\end{tabular}

a Common buckwheat (CB), tartary buckwheat (TB).

\section{Determination of rutin and quercetin}

HPLC analysis was carried out to determine the content of rutin and quercetin using an HPLC system (HITACHI, Japan) equipped with an L-2130 Pump fitted with an L-2200 Automatic Sampling machine. The analysis was performed on a C18 column $(4.6 \mathrm{~mm} \times 200 \mathrm{~mm}, 5 \mu \mathrm{m}$ particle size) (Dalian Elite YPERSIL, China). The mobile phase, a mixture of buffer $(0.4 \%$ phosphoric acid), and methanol $(50: 50 \mathrm{v} / \mathrm{v})$ was filtered through a $0.45 \mu \mathrm{m}$ membrane filter and degassed by sonication. HPLC analysis was performed at $30^{\circ} \mathrm{C}$ with a flow rate of $1.0 \mathrm{~mL} / \mathrm{min}$. The column effluent was monitored at $360 \mathrm{~nm}$. Quantification was performed by comparing the peak areas obtained from the samples with those of standards. Serial volumes $(1,2.5,5,10,15,20 \mu \mathrm{L})$ of 0.12 $\mathrm{mg} / \mathrm{mL}$ standard rutin solution and serial volumes $(3,5,10,15,20,25,30,35,40 \mu \mathrm{L})$ of $4 \times 10^{-3}$ $\mathrm{mg} / \mathrm{mL}$ standard quercetin solution were injected, respectively, for an HPLC analysis. The standard curve was developed with peak area as $\mathrm{y}$ and sample volume as $\mathrm{x}$.

\section{Validation}

The method was validated for precision, repeatability, stability, and accuracy. Instrument precision was checked by injecting $10 \mu \mathrm{L}$ farina sample \#1 solution three times and measuring the peak areas of rutin and quercetin, then the RSD (\%) was calculated. Repeatability was tested by preparing three samples farina extract \#6 (1.5 g per sample); the measurement according to the 
chromatography condition is mentioned above. Stability was studied by analyzing the contents of rutin and quercetin of sample \#8 farina extract on five different days, and calculating the RSD (\%) of daily averages. Accuracy of the method was studied using the method of standard addition. Standard rutin and quercetin solutions were added to the extract and the percent recovery was determined. The amounts of rutin and quercetin were determined and the percent recovery was calculated.

\section{Determination of amino acid}

Amino acid was determined according to the Amino Acid Quantitation Kit instructions. Briefly, the reaction system contained $0.5 \mathrm{~mL}$ sample, $1.0 \mathrm{~mL}$ reaction buffer, and $0.5 \mathrm{~mL}$ developer solution. The blank control and standard group were $0.5 \mathrm{~mL}$ distilled water and $0.5 \mathrm{~mL}$ standard amino acid solution, respectively, instead of $0.5 \mathrm{~mL}$ sample. The reaction system was mixed and centrifuged for $10 \mathrm{~min}\left(3500 \mathrm{rpm}^{-1}\right)$, and then the supernatant was measured for the absorption value at $630 \mathrm{~nm}$. The total amino acid content can be calculated by the equation from standard amino acid.

\section{Statistical analysis}

Data are reported as means \pm standard deviation (SD) for at least three replicates for each sample. Analysis of variance and least significant difference tests were conducted to identify differences among means using the SPSS 18.0 software. Correlation analyses were performed using a Pearson correlation test. Statistical significance was considered at $P<0.05$.

\section{RESULTS}

\section{Determination of rutin and quercetin in buckwheat farina and bran by HPLC}

\section{Chromatography conditions}

HPLC analysis was performed according to the following conditions: Dalian Elite YPERSIL $5 \mu \mathrm{m} 4.6$ x 200 mm C18 column (China), methonal-0.4 phosphoric acid water solution (50:50) as the mobile phase, with a detection wavelength of $360 \mathrm{~nm}$. Flow rate is $1.00 \mathrm{~mL} / \mathrm{min}$ and column temperature is $30^{\circ} \mathrm{C}$. The chromatography graphs of rutin and quercetin are shown in Figure 1 (A: standard, B: sample). Each peak in the sample was identified by comparing its retention time with that of the standard, which was carried out under the same chromatography conditions. The retention times of rutin and quercetin standards were 3.32 and 6.82 min respectively, while rutin and quercetin in samples were determined with retention times of 3.36 and $6.82 \mathrm{~min}$, respectively.
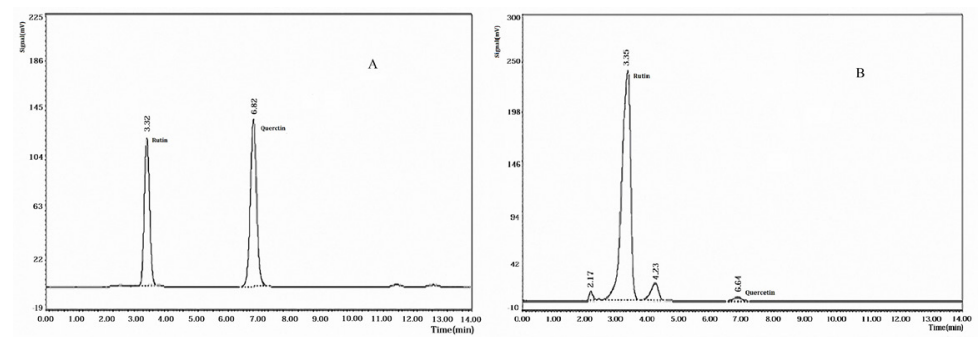

Figure.1 HPLC chromatograms of rutin and quercetin. A. Standard. B. Sample. 


\section{Method validation}

A linear relationship between the peak area and the amount of standard rutin was obtained for rutin in the range of $0.120-2.400 \mu \mathrm{g}$. The equation of linear regression curve obtained was $y$ $=1432.3 x-112.57$, where $y=$ (peak area), $x=$ ( volume of rutin), with a correlation coefficient $\left(r^{2}\right)$ of 0.9994 . The linearity range of standard quercetin was $0.012-0.160 \mu \mathrm{g}$ and the equation of linear regression was $y=1.9404 x+1.8326$, where $y=$ (peak area), $x=$ (volume of quercetin), with a correlation coefficient $\left(r^{2}\right)=0.9999$. The precision (RSD) was found to be $3.14 \%$ and the repeatability (RSD) of the method was $1.50 \%$. Values of inter-day precision (RSD) was found to be $3.28 \%$. The accuracy of the method was evaluated by recovery. The results of recovery analysis are shown in Table 2. The recovery of rutin and quercetin was 100.89 and $94.91 \%$, respectively, and the corresponding RSD was 1.97 and $0.26 \%$, respectively.

\begin{tabular}{|c|c|c|c|c|c|c|}
\hline & $\begin{array}{l}\text { Preanalyzed } \\
\text { sample }(\mathrm{mg})\end{array}$ & $\begin{array}{l}\text { Amount of standard to } \\
\text { preanalyzed sample }(\mathrm{mg})\end{array}$ & Total amount found & Recovery (\%) & Mean recovery (\%) & RSD (\%) \\
\hline \multirow{3}{*}{ Rutin } & 0.1095 & 0.2286 & 0.3256 & 94.53 & 94.91 & 1.97 \\
\hline & 0.1095 & 0.2286 & 0.3265 & 94.93 & & \\
\hline & 0.1095 & 0.2286 & 0.3273 & 95.28 & & \\
\hline \multirow[t]{3}{*}{ Quercetin } & 5.3378 & 7.5000 & 12.9240 & 101.15 & 100.89 & 0.26 \\
\hline & 5.3378 & 7.5000 & 12.7471 & 98.79 & & \\
\hline & 5.3378 & 7.5000 & 13.0433 & 102.74 & & \\
\hline
\end{tabular}

\section{Amounts of rutin and quercetin in buckwheat from different locations}

Samples from different locations are shown in Table 1. The rutin and quercetin contents of buckwheat seeds are shown in Figures 2 and 3. The rutin content ranged from $0.05 \%(0.05$ $\mathrm{g}$ per $100 \mathrm{~g}$ dry seeds) to $1.35 \%$. Obviously, tartary buckwheat seeds had higher rutin content than common buckwheat seeds (Figure 2). Similar difference in the flavonoid content was listed in the work of Pankaja et al. (2012). In their work, the sprouts, microgreens, and leafy greens of common and tartary buckwheat were compared for the phenolic contents. It was found that tartary buckwheat samples expressed higher total phenolic and flavonoid contents compared to the common buckwheat. Meanwhile, the bran had higher rutin content than the farina in tartary buckwheat seeds, with a respective content of $0.45-1.19 \%$ and $0.14-0.67 \%$. These results are consistent with the results of Cho et al. (2014). It was also found that buckwheat bran contained a distinctly higher content of rutin, compared to hull and flour. Tartary buckwheat from Sichuan (China) had the highest rutin content (1.35\%), followed by tartary buckwheat from Guizhou (China) out of the fourteen samples tested.

As Figure 3 shows, quercetin content was extremely low. No quercetin was detected in common buckwheat bran and farina. Quercetin was only found in some samples of tartary buckwheat and varied from 0.01 to $0.17 \%$. Similar results were recorded in the work of Ren and Sun (2014). In their work, no quercetin was detected in common buckwheat flour, bran, or sprouts from 1 to 9 days and quercetin was only found in tartary buckwheat sprouts during the early stages of germination. However, in the study of Kim et al. (2008), a trace of quercetin was found in nongerminated seeds while quercetin was not detected in the seed sprouts. It is important to emphasize that quantities of quercetin in buckwheat seeds were relatively low and close to the detection limit of HPLC, leading to the obscure observed results (Kim et al., 2004). Similar to rutin, it was found 
that bran contained higher amounts of quercetin than farina in tartary buckwheat. Concerning quercetin, the highest value $(0.17 \%)$ was determined in tartary buckwheat from Changzhi (Shanxi province, China) and varied from 0.01 to $0.17 \%$.

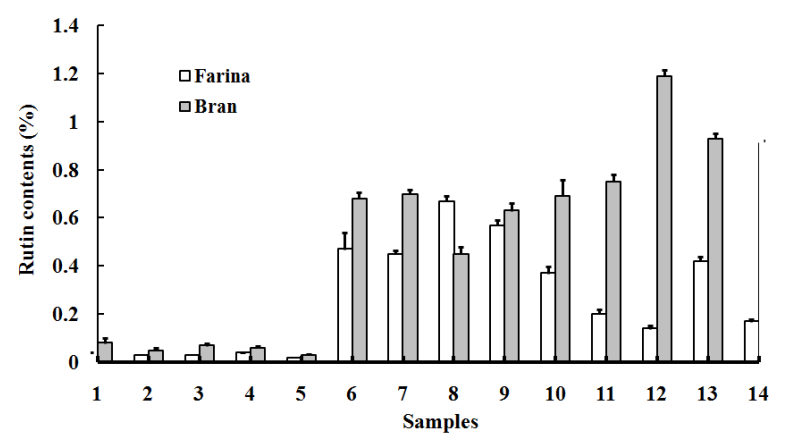

Figure 2. Rutin content of buckwheat collected from 14 locations determined in bran and farina.

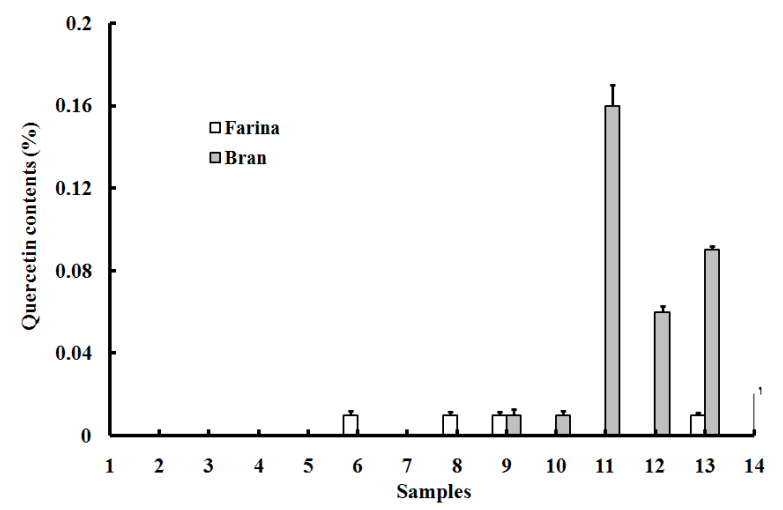

Figure 3. Quercetin content of buckwheat collected from 14 locations determined in bran and farina.

\section{Content of amino acids in buckwheat from different locations}

The content of amino acids is shown in Figure 4. As the results show, amino acid contents were around $0.18-1.27 \%$ in common buckwheat and $0.17-1.68 \%$ in tartary buckwheat seeds. Tartary buckwheat from Changzhi (Shanxi Province, China) had the highest amino acid content, followed by common buckwheat from Hebei (China).

Reported contents of amino acids varied greatly depending on the material sources. Additionally, different extraction and dilutions may have a significant impact on the relative quantities of amino acid (Mehdizadeh et al., 2015). In this study, the obtained results are in accordance with those of literature. According to Kivrak et al. (2014), the total free amino acids were $0.20 \%$ in the giant puffball mushroom (Calvatia gigantea) using UPLC-MS/MS. Content of free amino acids in hawthorns at different maturation stages was 0.32 to $0.35 \%$ ( $\mathrm{Li}$ et al., 2015). In the seeds of vegetable soybeans the amount of the free amino acids ranged from 0.46 to $1.02 \%$ (Song et al., 2013) and the contents of cocoa samples varied between 0.52 and $1.81 \%$ (Marseglia et al., 2014). 
Higher levels of free amino acid was detected in rambutan seed (9.73\%) and blueberry (18.7\%) (Zhang et al., 2014; Mehdizadeh et al., 2015).

More free amino acids were detected in bran than in farina in both common buckwheat and tartary buckwheat seeds in this study. These results are in accordance with literature. Different fractions and products of wheat, rye, oats, and barley were analyzed by Mustafa et al. (2007), and it was found that bran contained more free amino acids than did the other analyzed fractions of cereals.

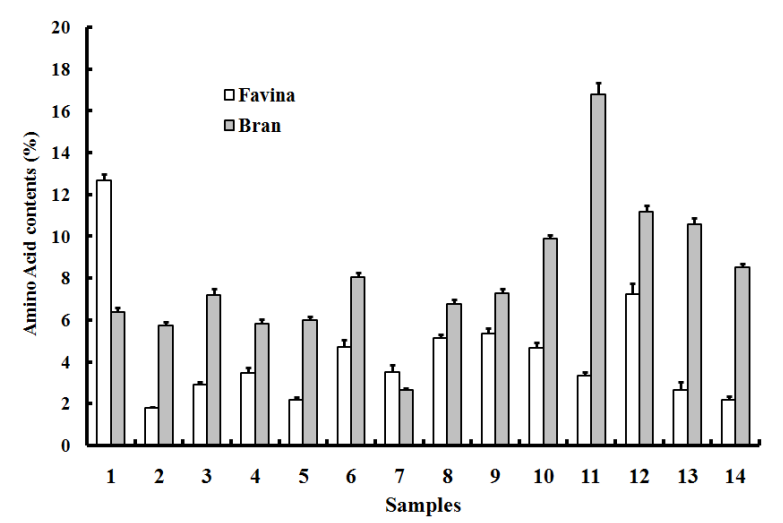

Figure 4. Amino acid content of buckwheat collected from 14 locations determined in bran and farina.

\section{DISCUSSION}

A precise and accurate HPLC method was established for determination of rutin and quercetin in buckwheat seeds. The method is fast, relatively economical, and can be applied to other grains. The results show significant variations in buckwheat seeds. The contents of rutin, quercetin, and amino acids in common buckwheat seeds were lower than those in tartary buckwheat seeds. Quercetin could not be detected in some samples of common buckwheat in this study. The relatively high rutin content in both common and tartary buckwheat implies that buckwheat may serve as an excellent dietary source of rutin, which is known to have beneficial health effects such as reduced blood pressure, lowered blood sugar concentration, and increased antioxidant activity (Hernández-Herrero and Frutos, 2015).

The bran has higher rutin, quercetin, and amino acids than the farina in common and tartary buckwheat, suggesting that food products made of whole-buckwheat flour is more healthful than that of fine white flour. However, the texture of whole-wheat flour food is usually not as desirable. Therefore, it is necessary to improve the processing methods of buckwheat foods to meet the demand for both texture and nutritional value. Furthermore, our results highlight the possibility of using buckwheat bran, a by-product of buckwheat flour production, as a source of rutin for both dietetic and industrial application.

\section{ACKNOWLEDGMENTS}

We are grateful to Yan Chai and Peng-Ke Wang for providing tartary buckwheat samples and Yu Tang for excellent technical assistance. Research supported by the Natural Sciences Foundation of China (\#31300653 and \#31171659). 


\section{REFERENCES}

Cho YJ, Bae IY, Nglett GE and Lee S (2014). Utilization of tartary buckwheat bran as a source of rutin and its effect on the rheological and antioxidant properties of wheat-based products. Ind. Crops and Prod. 61: 211-216.

Danihelová E and Kalinova J (2010). Determination of quercetin glycosides and free quercetin in buckwheat by capillary micellar electrokinetic chromatography. J. Separat. Sci. 33: 1633-1138.

Elfalleh W, Hannachi H, Guetat A, Tlili N, et al. (2012). Storage protein and amino acid contents of Tunisian and Chinese pomegranate (Punica granatum L.) cultivars. Genet. Resour. Crop Evol. 59: 999-1014.

Gunaratne A, Wu K, Li D, Bentota A, et al. (2013). Antioxidant activity and nutritional quality of traditional red-grained rice varieties containing proanthocyanidins. Food Chem. 138: 1153-1161.

Guo XD, Ma YJ, Parry J, Gao JM, et al. (2011). Phenolics content and antioxidant activity of tartary buckwheat from different locations. Molecules 16: 9850-9867.

Hernández-Herrero JA and Frutos MJ (2015). Influence of rutin and ascorbic acid in colour, plum anthocyanins and antioxidant capacity stability in model juices. Food Chem. 173: 495-500.

Horbowicz M and Obendorf RL (1992). Changes in sterols and fatty acids of buckwheat endosperm and embryo during seed development. J. Agr. Food Chem. 40: 745-750.

Jiang P, Burczynski F, Campbell C, Pierce G, et al. (2007). Rutin and flavonoid contents in three buckwheat species Fagopyrum esculentum, F. tataricum, and F. homotropicum and their protective effects against lipid peroxidation. Food Res. Int. 40: 356-364.

Joanna JG, Langner E and Rzeski W (2011). Kinetic studies of the effects of Temodal and quercetin on astrocytoma cells. Pharmacol. Rep. 63: 403-416.

Kim SL, Kim SK and Park CH (2004). Introduction and nutritional evaluation of buckwheat sprouts as a new vegetable. Food Res. Int. 37: 319-327.

KIvrak I, KIvrak S and Harmandar M (2014). Free amino acid profiling in the giant puffball mushroom (Calvatia gigantea) using UPLC-MS/MS. Food Chem. 158: 88-92.

Li WQ, Hu QP and Xu JG (2015). Changes in physicochemical characteristics and free amino acids of hawthorn (Crataegus pinnatifida) fruits during maturation. Food Chem. 175: 50-56.

Marseglia A, Palla G and Caligiani A (2014). Presence and variation of $y$-aminobutyric acid and other free amino acids in cocoa beans from different geographical origins. Food Res. Int. 63: 360-366.

Martina D and Ernest S (2012). Nutritional and health benefits of buckwheat. Potravinarstvo 6: 1-9.

Matsui T, Kudo A, Tokuda S, Matsumoto K, et al. (2010). Identification of a new natural vasorelaxatant compound, (+)-osbeckic acid, from rutin-free tartary buckwheat extract. J. Agric. Food Chem. 58: 10876-10879.

Mehdizadeh S, Lasekan O, Muhammad K and Baharin B (2015). Variability in the fermentation index, polyphenols and amino acids of seeds of rambutan (Nephelium lappaceum L.) during fermentation. J. Food Comp. Anal. 37: 128-135.

Mustafa A, Aman P, Andersson R, Afaf K, et al. (2007). Analysis of free amino acids in cereal products. Food Chem. 105: 317-324.

Pankaja S, Amal KG, Anup G, Cheng WJ, et al. (2012). Phenolic contents, antioxidant and a-glucosidase inhibition properties of Nepalese strain buckwheat vegetables. Afr. J. Biotechnol. 11: 184-190.

Pratta GR, Rodríguez GR, Zorzoli R, Picardi LA, et al. (2011). Biodiversity in a tomato germplasm for free amino acid and pigment content of ripening fruits. Am. J. Plant Sci. 2: 255-261.

Pugliese AG, Tomas-Barberan FA, Truchado P and Genovese Ml (2013). Flavonoids, proanthocyanidins, vitamin C, and antioxidant activity of Theobroma grandiflorum (Cupuassu) Pulp and Seeds. J. Agric. Food Chem. 61: 2720-2728.

Ren SC and Sun JT (2014). Changes in phenolic content, phenylalanine ammonia-lyase (PAL) activity, and antioxidant capacity of two buckwheat sprouts in relation to germination. J. Func. Foods 7: 298-304

Song JF, Liu CQ, Li DJ and Gu ZX (2013). Evaluation of sugar, free amino acid, and organic acid compositions of different varieties of vegetable soybean (Glycine max [L.] Merr). Ind. Crops and Prod. 50: 743-749.

Steadman KJ, Burgoon MS, Lewis BA, Edwardson SE, et al. (2001a). Buckwheat seed milling fractions: description, macronutrient composition, and dietary fiber. J. Cereal Sci. 33: 271-278.

Steadman KJ, Burgoon MS, Lewis BA, Edwardson SE, et al. (2001b). Minerals, phytic acid, tannin and rutin in buckwheat seed milling fractions. J. Sci. Food Agric. 81: 1094-1100.

Tomotake H, Shimaoka I, Kayashita J, Yokoyama F, et al. (2000). A buckwheat protein product suppresses gallstone formation and plasma cholesterol more strongly than soy protein isolate in hamsters. J. Nutr. 130: 1670-1674.

Wang G, Wang JJ, Yang GY, Du SM, et al. (2012). Effects of quercetin nanoliposomes on C6 glioma cells through induction of type III programmed cell death. Int. J. Nanomed. 7: 271-280. 
Xiao ZP (2003). Prospect of development and exploitation of buckwheat resources. Modern Agric. 8: 35.

Zhang H, Wang ZY, Yang X, Zhao HT, et al. (2014). Determination of free amino acids and 18 elements in freeze-dried strawberry and blueberry fruit using an Amino Acid Analyzer and ICP-MS with micro-wave digestion. Food Chem. 147: 189-194.

Zhang R, Zeng Q, Deng Y, Zhang M, et al. (2013). Phenolic profiles and antioxidant activity of litchi pulp of different cultivars cultivated in Southern China. Food Chem. 136: 1169-1176.

Zhang ZL, Zhou ML, Tang Y, Li FL, et al. (2012). Bioactive compounds in functional buckwheat food. Food Res. Int. 49: 389-395.

Zou YX, Liao ST, Shen WZ, Liu F, et al. (2012). Phenolics and antioxidant activity of mulberry leaves depend on cultivar and harvest month in southern China. Int. J. Mol. Sci. 13: 16544-16553. 